\title{
Maternal serum levels of perfluoroalkyl substances and organochlorines and indices of fetal growth: a Scandinavian case-cohort study
}

Hilde B. Lauritzen', Tricia L. Larose' ${ }^{1}$, Torbjørn Øien', Torkjel M. Sandanger ${ }^{2,3}$, Jon Ø. Odland ${ }^{2,4}$, Margot van de Bor ${ }^{5}$ and Geir W. Jacobsen'

BACKGROUND: The associations between prenatal exposure to endocrine disruptive chemicals (EDCs) and fetal growth are inconsistent, and few studies have considered small-for-gestational-age (SGA) birth as an outcome. Our current study of Scandinavian parous women aimed to address these inconsistencies and gaps in the literature.

METHODS: This case-cohort study included 424 motherchild pairs who participated in a prospective, multi-center study of parous women in Norway (Trondheim and Bergen) and Sweden (Uppsala). We used linear and logistic regression with 95\% confidence intervals (Cls) to analyze the associations between two perfluoroalkyl substances (PFASs) and five organochlorines (OCs) from early second trimester and indices of fetal growth.

RESULTS: Among Swedish women, prenatal exposure to perfluorooctanoate (PFOA), polychlorinated biphenyl (PCB) 153 and hexachlorobenzene (HCB) were associated with higher odds for SGA birth. We found stronger associations among Swedish male offspring. In the Norwegian cohort, we found no significant associations between EDC exposure and indices of fetal growth.

CONCLUSIONS: Some populations may be more vulnerable to EDCs, possibly due to differences in exposure levels, exposure sources and/or modifiable lifestyle factors. Male offspring may be more vulnerable to endocrine disruption.

cetal etal growth restriction (FGR) is defined as a pathologic inhibition of intrauterine fetal growth and failure to achieve the fetus' growth potential. FGR is associated with perinatal mortality and morbidity. Small-for-gestational-age (SGA) is a proxy for FGR and defined as birth weight below the 10th percentile for gestational age, sex, and parity (1).

Perfluoroalkyl substances (PFASs) and organochlorines (OCs) are persistent, bio-accumulative chemicals that have been detected in maternal blood during pregnancy and in cord blood at delivery. PFASs and OCs may act as endocrine disrupting chemicals (EDCs), and in utero exposure to EDCs may have consequential developmental effects on the fetus (2). A United Nations Environment Program/World Health Organization (UNEP/WHO) report from 2012 identifies human exposure to EDCs as an emerging challenge (3). Increased knowledge is required to ensure better protection for the most vulnerable members of society. Pregnant mothers and children are particularly vulnerable to developmental exposures like EDCs, and fetal programming may increase susceptibility to diseases later in life (3).

The associations between EDCs and indices of fetal growth have been studied in different populations, although results are inconsistent $(4,5)$. Diet is considered an important exposure route for PFASs and OCs even in periods with simultaneous direct exposure from production and emission of chemicals (6). In Scandinavian populations, consumption of seafood have been associated with high PFAS and OC serum levels $(7,8)$. The Baltic Sea, which is situated on the East coast of Sweden, has been vastly contaminated with OCs (9), and a study of births in Sweden from 1973-1991 found associations between high maternal intake of fish from the Baltic Sea and restricted fetal growth (10). However, most other studies report positive associations between higher maternal fish intake and better fetal growth (11). Dietary intake of fish from different water sources may result in population-specific EDC exposures. Taken together, source-specific fish intake may confound associations between EDCs and fetal growth.

Most observational studies have been underpowered to detect significant associations between EDCs and SGA birth, or only included birth weight as an outcome. A most recent systematic review of prenatal serum levels of PFASs and human fetal growth published in 2015, recommended that future EDC exposure studies should focus on SGA birth as an outcome of interest (4).

In the current study, we used a case-cohort design to study the association between maternal serum levels of PFASs and OCs and indices of fetal growth including birth weight, birth

\footnotetext{
'Department of Public Health and General Practice, Norwegian University for Science and Technology, Trondheim, Norway; ${ }^{2}$ Department of Community Medicine, University of Troms $\varnothing$ - The Arctic University of Norway, Troms $\varnothing$, Norway; ${ }^{3}$ NILU-Norwegian Institute for Air Research, Fram High north research Centre, Troms $\varnothing$, Norway; ${ }^{4}$ School of Health Systems and Public Health, University of Pretoria, Pretoria, South Africa; ${ }^{5}$ Section of Health and Life Sciences, Vrije Universiteit, Amsterdam, The Netherlands. Correspondence: Hilde B. Lauritzen (hilde.b.lauritzen@ntnu.no)

Received 23 March 2016; accepted 8 August 2016; advance online publication 26 October 2016. doi:10.1038/pr.2016.187
} 
length, head circumference, and SGA birth in Scandinavian women from Norway and Sweden. We also explored possible effect modification by country and offspring sex.

\section{RESULTS}

\section{Maternal and Offspring Baseline Characteristics}

Overall, Swedish women had, on average, higher prepregnancy BMI (22 vs. $\left.21 \mathrm{~kg} / \mathrm{m}^{2}\right)$ compared to Norwegian women. A greater proportion of Swedish women were nonsmokers at the time of conception compared to their Norwegian peers (67 vs. $47 \%$ nonsmokers) (Table 1). The total cohort of Swedish mothers compared to Norwegian mothers did not differ significantly in other baseline characteristics (maternal age, height, education, parity, and interpregnancy interval). Swedish offspring were on average longer at birth compared to Norwegian offspring, but did not differ significantly in other characteristics (birth weight, head circumference, and gestational age).

Swedish women who gave birth to SGA offspring (SGA mothers) were on average shorter, had lower prepregnancy BMI, fewer years of education, and lower parity compared to Swedish women with non-SGA births (non-SGA mothers). A higher proportion of Swedish SGA mothers reported smoking at conception compared to Swedish non-SGA mothers (60 vs. $21 \%$ smokers). We observed a similar pattern in baseline characteristics when Norwegian SGA and non-SGA mothers were compared. Norwegian SGA mothers were also significantly younger than Norwegian non-SGA mothers (28 vs. 29 $y$ of age). Swedish SGA offspring had, on average, lower birth weight, shorter birth length and smaller head circumference compared to Swedish non-SGA offspring. Norwegian SGA offspring had in addition significantly lower gestational age compared to the Norwegian non-SGA offspring.

\section{PFAS and OC Levels}

Overall, Swedish mothers had significantly higher median serum levels of PFOA (2.33 vs. $1.62 \mathrm{ng} / \mathrm{ml}$ ), perfluoroctane sulfonate (PFOS) (16.4 vs. $9.74 \mathrm{ng} / \mathrm{ml}$ ), PCB 153 (117 vs. $80.1 \mathrm{ng} / \mathrm{g}$ lipid), and $\beta-\mathrm{HCH}$ (25.0 vs. $21.2 \mathrm{ng} / \mathrm{g}$ lipid) compared to the total Norwegian cohort (Table 2, Figure 1). Swedish mothers had significantly lower median serum levels of $t$-NC (6.28 vs. $6.74 \mathrm{ng} / \mathrm{g}$ lipid) compared to Norwegian mothers (Table 2, Figure 1).

Median levels of PFOA and PCB 153 were significantly higher among Swedish SGA mothers compared to Swedish non-SGA mothers (Figure 1). Differences in levels of other EDCs (PFOS, $p, p^{\prime}$-DDE, HCB, $\beta$ - $\mathrm{HCH}$ and $t$-NC) were not statistically significant when Swedish SGA mothers and Swedish non-SGA mothers were compared (Figure 1).

We observed no significant differences in PFAS or OC levels between Norwegian SGA mothers and Norwegian non-SGA mothers.

Overall, there were medium to high correlations within the PFASs $(\rho=0.56-0.73)$ and within the OCs $(\rho=0.29-0.70)$ (Supplementary Figure S1 online). However, the PFASs were not highly correlated with the OCs $(\rho=-0.01-0.22)$
(Supplementary Figure S1 online). The same pattern was observed in both Norwegian and Swedish cohorts.

\section{Associations Between PFAS and OC Levels and Indices of Fetal Growth}

In the pooled analyses including data from both Norway and Sweden, we found no significant associations between EDCs and indices of fetal growth after adjustment for important covariates (Supplementary Table S1 online).

Table 3 shows associations between serum levels of PFASs and OCs and indices of fetal growth stratified by country of residence. In adjusted linear models for the Swedish cohort, birth weight decreased significantly by $-359 \mathrm{~g}(95 \% \mathrm{CI}:-596$, $-122)$ and $-292 \mathrm{~g}(95 \% \mathrm{CI}:-500,-84)$ per ln-unit increase in PFOA and PFOS, respectively. Birth length was also negatively associated with increasing levels of PFOA and PFOS. For each ln-unit increase in HCB levels, a significant decrease in head circumference $(\beta=-1.0 \mathrm{~cm}(95 \% \mathrm{CI}:-1.7,-0.2))$ was observed after adjustment.

In multivariate logistic regression models for the Swedish cohort, we observed a significant increase in adjusted odds for SGA birth per ln-unit increase in PFOA (aOR = 5.25 (95\% CI: 1.68-16.4)), PCB 153 (aOR = 5.59 (95\% CI: 1.05-29.9)) and $\mathrm{HCB}(\mathrm{aOR}=5.62$ (95\% CI: 1.26-25.1)). We also observed increased aOR for SGA birth per ln-unit increase in PFOS $(\mathrm{aOR}=2.51$ (95\% CI: 0.93-6.77)), although nonsignificant. Among Swedish women, there were no significant associations between levels of PFASs or OCs and gestational age (Table 3). Among Norwegian women, we observed no statistically significant associations between PFASs or OCs and indices of fetal growth (Table 3).

In the Swedish cohort, the $P$ value for interaction between PFOA and infant sex was 0.046 in multivariate linear regression models with birth weight as an outcome. No other interaction terms between EDCs and infant sex were statistically significant $(P<0.10)$ (data not shown). In Swedish male offspring, we observed a significant decrease in birth weight $(\beta=$ $-526 \mathrm{~g} ; 95 \% \mathrm{CI}:-828,-222)$ and birth length $(\beta=-1.6 \mathrm{~cm}$; 95\% CI: -2.9, -0.4) for each ln-unit increase in PFOA (Table 4 and Figure 2). We also observed a significant increase in adjusted odds for SGA birth (aOR $=6.55$; 95\% CI: 1.14-37.5) among male Swedish offspring (Table 4 and Figure 2). No statistically significant associations between PFOA and indices of fetal growth were observed among Swedish female offspring. We found no significant interaction terms between PFASs or OCs and offspring sex in the Norwegian cohort (data not shown).

\section{DISCUSSION}

In this case-cohort study of 424 Scandinavian parous women, we observed increased odds of SGA birth for each ln-unit increase in PFOA, PCB 153 and HCB, among Swedish but not Norwegian mothers. Increasing levels of PFOA, PFOS and HCB were negatively associated with other indices of fetal growth including birth weight, birth length and head circumference among the Swedish mothers. The associations 
Table 1. Maternal and offspring baseline characteristics by country and SGA status

\begin{tabular}{|c|c|c|c|c|c|c|}
\hline & \multicolumn{3}{|c|}{ Norway (Trondheim: $N=137$, Bergen: $N=128$ ) } & \multicolumn{3}{|c|}{ Sweden (Uppsala: $N=159$ ) } \\
\hline & Non-SGA $(N=174)$ & $\mathrm{SGA}(N=91)$ & Total $(N=265)$ & Non-SGA $(N=107)$ & $\mathrm{SGA}(N=52)$ & Total $(N=159)$ \\
\hline & $\begin{array}{l}\text { Mean }(95 \% \mathrm{Cl}) \\
\quad \text { or } n(\%)\end{array}$ & $\begin{array}{l}\text { Mean }(95 \% \mathrm{Cl}) \\
\quad \text { or } n(\%)\end{array}$ & $\begin{array}{l}\text { Mean }(95 \% \mathrm{Cl}) \\
\quad \text { or } n(\%)\end{array}$ & $\begin{array}{l}\text { Mean }(95 \% \mathrm{Cl}) \\
\quad \text { or } n(\%)\end{array}$ & $\begin{array}{l}\text { Mean }(95 \% \mathrm{Cl}) \\
\text { or } n(\%)\end{array}$ & $\begin{array}{l}\text { Mean }(95 \% \mathrm{Cl}) \\
\quad \text { or } n(\%)\end{array}$ \\
\hline \multicolumn{7}{|l|}{ Maternal characteristics } \\
\hline High-risk group & $0(0)$ & $74(81)^{*}$ & $74(28)$ & $0(0)$ & $43(83)^{*}$ & $43(27)$ \\
\hline Maternal height & $167.0(166.1-167.9)$ & $164.3(163.2-165.4)^{*}$ & $166.1(165.3-166.8)$ & $166.6(165.5-167.7)$ & $164.2(162.7-165.7)^{*}$ & $165.8(164.9-166.7)$ \\
\hline $\begin{array}{l}\text { Maternal prepregnancy } \\
\text { BMI }\end{array}$ & $21.6(21.2-22.0)$ & $20.2(19.7-20.7)^{*}$ & $21.120 .8-21.4)$ & $22.6(22.1-23.1)$ & $20.9(20.2-21.6)^{*}$ & $22.1(21.6-22.5)^{*}$ \\
\hline \multicolumn{7}{|l|}{ Education (years) } \\
\hline \multicolumn{7}{|c|}{ Smoking at conception (number of cigarettes) } \\
\hline 0 & $103(59)$ & $20(22)^{*}$ & $123(47)$ & $85(79)$ & $21(40)^{*}$ & $106(67)^{*}$ \\
\hline $1-9$ & $21(12)$ & $14(16)$ & $35(13)$ & $4(3.7)$ & $3(5.8)$ & $7(4.4)$ \\
\hline 10 or more & $50(29)$ & $56(62)^{*}$ & $106(40)$ & $18(17)$ & $28(54)^{*}$ & $46(29)$ \\
\hline \multicolumn{7}{|l|}{ Parity } \\
\hline 1 & $122(70)$ & $65(71)$ & $186(71)$ & $66(62)$ & $39(75)^{*}$ & $104(66)$ \\
\hline 2 & $52(30)$ & $26(29)$ & $78(29)$ & $41(38)$ & $13(25)$ & $54(34)$ \\
\hline \multicolumn{7}{|c|}{ Interpregnancy interval (months) } \\
\hline Female & $87(50)$ & $44(48)$ & $131(50)$ & $55(51)$ & $23(44)$ & $77(49)$ \\
\hline Weight (g) & $3661(3590-3732)$ & $2882(2815-2949)^{*}$ & $3402(3335-3469)$ & $3790(3680-3900)$ & $2891(2820-2963)^{*}$ & $3503(3403-3601)$ \\
\hline Length $(\mathrm{cm})$ & $50.8(50.5-51.1)$ & $48.3(47.9-48.8)^{*}$ & $50.0(49.8-50.3)$ & $51.4(50.9-51.9)$ & $48.4(48.0-48.8)^{*}$ & $50.5(50.1-50.8)^{*}$ \\
\hline $\begin{array}{l}\text { Head circumference } \\
(\mathrm{cm})\end{array}$ & $35.4(35.2-35.5)$ & $34.0(33.7-34.2)^{*}$ & $34.9(34.7-35.1)$ & $35.5(35.2-35.8)$ & $33.7(33.4-33.9)^{*}$ & $34.9(34.7-35.1)$ \\
\hline Gestational age & $40.0(39.8-40.2)$ & $39.7(39.4-40.0)$ & $39.9(39.8-40.1)$ & $40.2(40.0-40.6)$ & $39.5(39.1-39.9)^{*}$ & $40.1(39.9-40.3)$ \\
\hline
\end{tabular}

*Total group: Significant difference between the total group in Norway vs. the total group in Sweden $(P<0.05)$ using Student $t$-test for normally distributed variables and Mann-Whitney U-test for categorical variables. SGA-group: Significant difference between SGA vs. non-SGA group within country strata $(P<0.05)$ using Student $t$-test for normally distributed variables and Mann-Whitney $U$-test for categorical variables.

SGA, small for gestational age.

between PFOA and SGA birth, birth weight, birth height and head circumference were stronger among male offspring in the Swedish cohort. We found no statistically significant associations between EDCs and indices of fetal growth among Norwegian women in this study population.

Birth weight, adjusted for gestational age at birth, is often used as a proxy for fetal growth in observational studies. However, adjusting for gestational age, which acts like a mediator in the association between birth weight and health outcomes, may introduce bias (12). SGA birth is a better marker for fetal growth restriction because it identifies babies with low birth weight accounting for the gestational age at birth. Only four studies have assessed associations between PFASs and SGA birth (13-16) with conflicting results. One study found increased odds for SGA with higher PFOS (13), and none found statistically significant associations with 


\section{Articles Lauritzen etal.}

Table 2. Wet-weight levels of PFASs, and wet-weight and lipid-adjusted levels of OCs in serum by country from the study population in the SGA-study, $N=424$

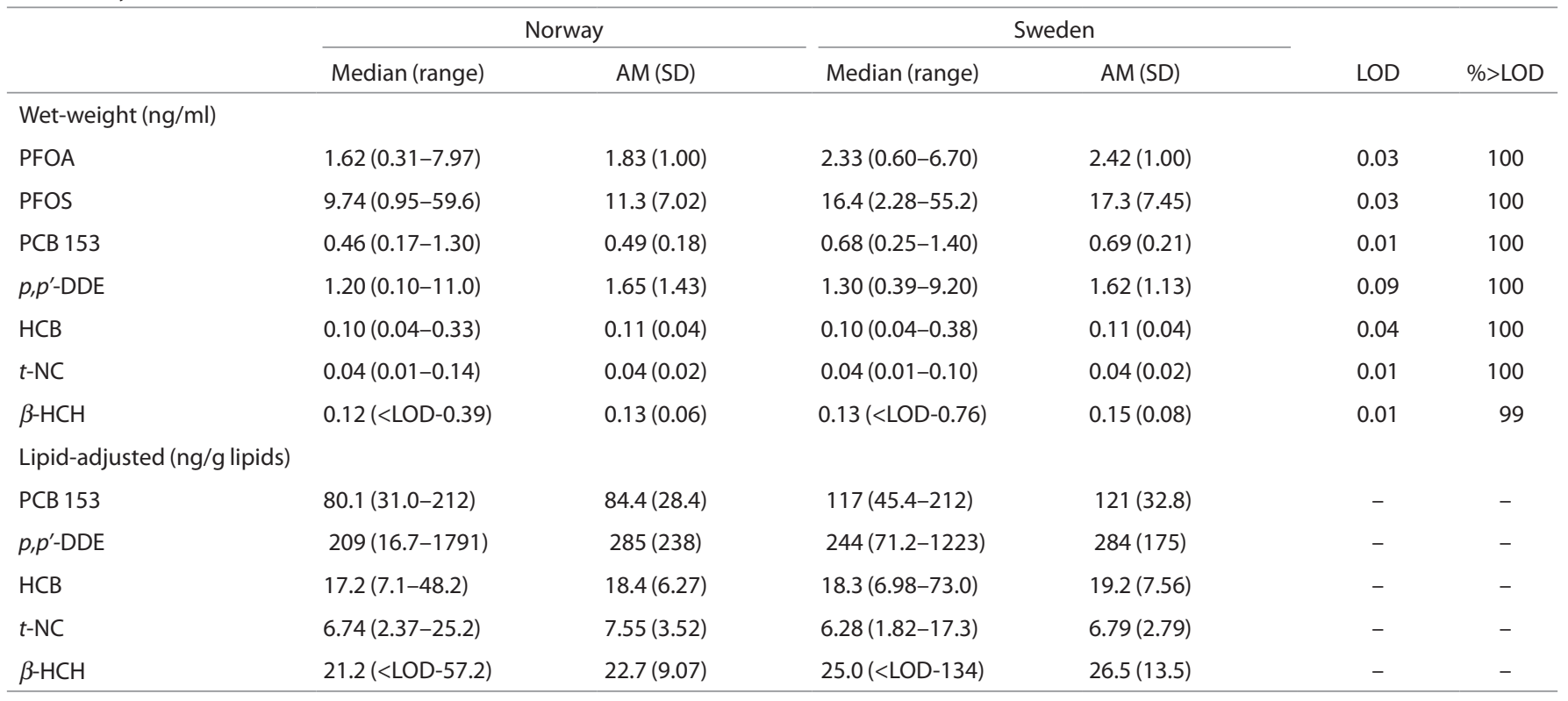

AM, arithmetic mean; HCB, hexachlorobenzene; LOD, limit of detection; \%>LOD, percentage of samples over limit of detection. Levels below $L O D$ were set to LOD/ $\sqrt{2} ; \mathrm{PCB}$, polychlorinated biphenyl; PFOA, perfluorooctanoate; PFOS, perfluoroctane sulfonate.

PFOA. In contrast, we observed increased odds for SGA birth with increasing levels of PFOA in our Swedish study population. Our finding of increased odds for SGA birth with increasing levels of PCB 153 in the Swedish cohort has not previously been reported, but is consistent with a recent meta-analysis that established an association between PCB 153 and birth weight (5). The increased odds for SGA birth with increasing levels of HCB among our Swedish women has not been established in previous studies.

The possible biological mechanisms for the effects of PFASs and OCs on fetal growth are uncertain, but their endocrinedisruptive properties may be involved. Normal development is highly dependent on thyroid and sex steroid hormones, and the prenatal period is a critical period for hormonal changes. Thyroid hormones are important for somatic growth and are involved in differentiation and functions of target tissues during development (17). Prenatal estrogens are also important in promoting fetal growth (18). Both PFASs and OCs have been associated with lower levels of circulating thyroid hormones $(19,20)$. Competitive binding to estrogenic receptors, disruption of enzymes or inhibition of the effects of endogenous estrogens are hypothesized actions of estrogenic and antiestrogenic PCB congeners, and may lead to inhibition of fetal growth (21). Moreover, human in vitro studies have found that PFASs interfere with the estrogen and androgen receptor (22). Since sex steroids might be disrupted by PFASs and OCs, there could be different effects in male and female fetuses which may explain our findings of stronger negative associations between PFOA and indices of restricted fetal growth among male but not female offspring in the Swedish cohort. Our sex specific finding differs from a Japanese study from 2009 that showed stronger negative associations between PFOS and birth weight among girls ( $n=428$ mother-child pairs) (23). The biological mechanisms for the sex difference in the toxicity for PFOA remains to be established, and it is possible that decreasing the sample size by stratifying our groups by sex reduced the precision of our results. Thus, further studies are warranted to elucidate any sex-related differences in fetal growth indices based on in utero exposure to PFASs.

The conflicting estimates on fetal growth between the Norwegian and Swedish cohort may be explained by differences in exposure levels and heterogeneity in exposure routes. Seafood and particularly fatty fish have been associated with high PFAS and OC levels in Scandinavian populations $(7,8)$. In our study, Norwegian women with fish consumption $>50 \mathrm{~g} / \mathrm{d}$ in the second trimester had 20 and $19 \%$ higher PFOA and PFOS levels, respectively, compared to women who did not eat fish (Supplementary Table S2 online). However, other food items, like meat, may be more important in other populations with less fish intake (24). Moreover, we cannot discount the possibility of differences in dietary patterns between Scandinavian countries, which may partially account for our results. Since fish consumption is associated with both EDC levels and fetal growth, this may introduce bias in epidemiological studies. A recent report from the Norwegian Scientific Committee for Food Safety concluded that overall, there is a beneficial effect of fish consumption on birth weight, because fatty acids and vitamins important for enhancing fetal growth outweigh potential negative effects from EDCs (11). However, it is important to note that this evaluation was based on evidence from studies conducted when EDC levels were lower than the EDC levels found during our study period (1986-88). Our estimates in the Norwegian cohort did not substantially change when we adjusted for fish consumption (data not 
a

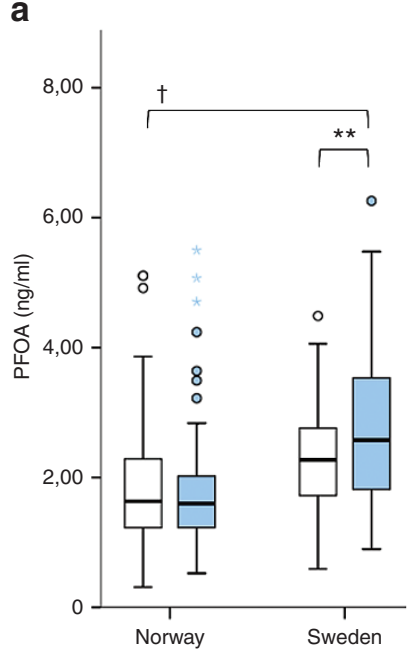

e

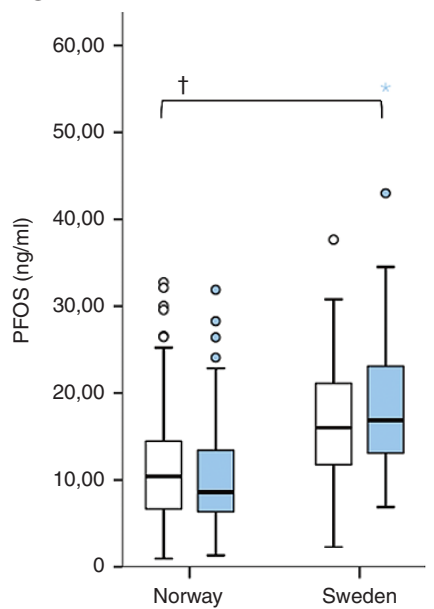

b

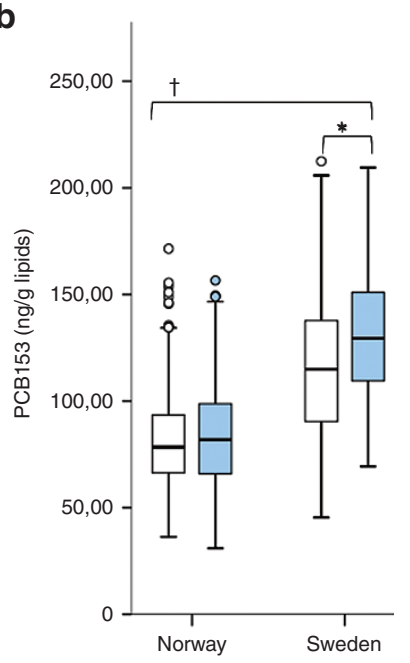

f

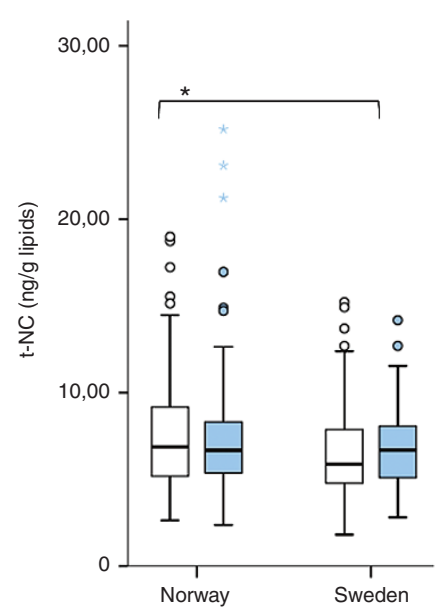

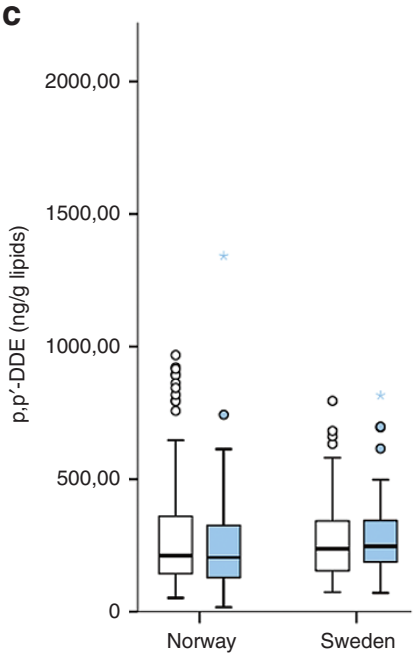

g

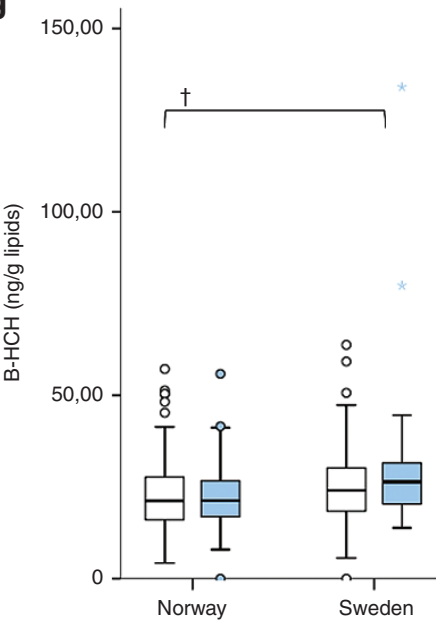

d

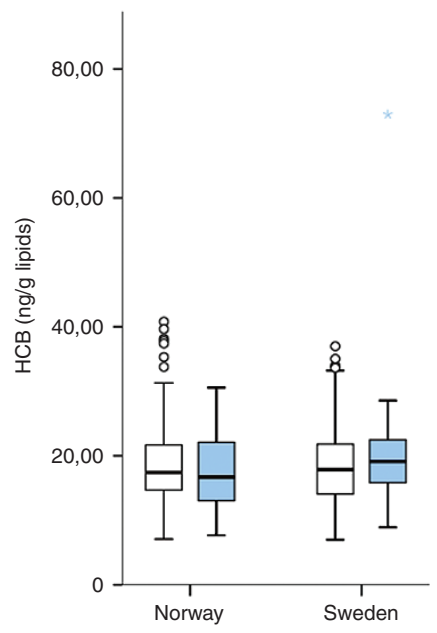

Figure 1. Levels of perfluoroalkyl substances ( $\mathrm{ng} / \mathrm{ml}$ ) and organochlorines (ng/g lipids) analyzed in maternal serum in second trimester, stratified by country of residence and SGA status (a: PFOA, b: PCB 153, c: $p, p^{\prime}-D D E, \mathbf{d}$ : HCB, e: PFOS, f: $t$-NC, g: $\beta$-HCH). White boxes: non-SGA mothers, light blue boxes: SGA-mothers. Boxes represent the 25 th-75th percentiles, horizontal lines represents the median, whiskers indicate 1.5 times the length of the interquartile range above and below the 75th and 25 th percentiles, respectively, and outliers are represented as data points. ${ }^{*} P \leq 0.05$, ${ }^{*} P \leq 0.01$, and $+P \leq 0.001$ for comparisons of levels between countries and between SGAs and non-SGAs within each country. HCB, hexachlorobenzene; PCB, polychlorinated biphenyl; PFOA, perfluorooctanoate; PFOS, perfluoroctane sulfonate; SGA, small for gestational age.

shown). Seasonal or daily variations in dietary intake of fish may introduce differential misclassification bias when using $3 \mathrm{~d}$ dietary intake as a measure of the overall consumption pattern, and we may not be able to capture the "real" fish eaters by this approach. We did not have information on fish consumption in the Swedish cohort.

In addition to different dietary patterns between populations and temporal trends of EDCs, there might be differences in contamination levels between fish sources. Studies have shown high OC contamination of the fish in the Baltic Sea on the East Coast of Sweden (9). Another study (1973-1991) that compared fishermen's pregnant wives from the East and West coast of Sweden, found that higher fish consumption from the East Coast resulted in higher maternal serum levels of PCB 153, which again resulted in increased risk of having an infant with low birth weight (25).

Smoking is often closely associated with socio-economic, dietary and lifestyle variables. In the Swedish cohort, smokers had 20\% higher PFOA levels compared to nonsmokers (Supplementary Table S2 online). Whereas, in the Norwegian cohort, smokers had 19\% lower PFOS levels compared to nonsmokers. The fact that PFASs were higher among smokers in Sweden, but lower among smokers in Norway, speaks against a potential direct biologic effect of smoking on PFAS levels. In addition, we found no evidence of stronger associations between PFASs and fetal growth among the smokers compared to the non-smokers in Norway or Sweden, respectively. Taken together, this suggests that there might be differences in socio-economic status, lifestyle, and dietary variables between the women with high PFAS levels in Norway and Sweden, respectively, which will further confound the analyses. Problems with individual and local differences in EDC levels will be greater during time periods with continued use of contaminants, and possible contamination of the drinking water with PFASs in Sweden (26) may account 
Articles | Lauritzen etal.

Table 3. Beta coefficients ( $\beta$ ) and odds ratios (ORs) with $95 \%$ confidence intervals ( $95 \% \mathrm{Cl}$ ) for associations between PFAS and organochlorines and indices of fetal growth (Norway: $N=265$, Sweden: $N=159$ )

\begin{tabular}{|c|c|c|c|c|c|c|c|c|c|c|}
\hline & \multicolumn{2}{|c|}{ Birth weight $(\mathrm{g})^{a}$} & \multicolumn{2}{|c|}{ Birth length $(\mathrm{cm})^{\mathrm{a}}$} & \multicolumn{2}{|c|}{ Head circumference $^{\mathrm{a}}(\mathrm{cm})$} & \multicolumn{2}{|c|}{ Gestational age $^{a}$ (weeks) } & \multicolumn{2}{|l|}{$S G A^{b}$} \\
\hline & $\beta(95 \% \mathrm{Cl})$ & $P$ & $\beta(95 \% \mathrm{Cl})$ & $P$ & $\beta(95 \% \mathrm{Cl})$ & $P$ & $\beta(95 \% \mathrm{Cl})$ & $P$ & OR $(95 \% \mathrm{Cl})$ & $P$ \\
\hline \multicolumn{11}{|l|}{ Norway } \\
\hline PFOA & $37(-99,174)$ & 0.590 & $-0.1(-0.7,0.4)$ & 0.656 & $0.2(-0.2,0.5)$ & 0.354 & $-0.2(-0.6,0.2)$ & 0.431 & $0.66(0.33-1.33)$ & 0.246 \\
\hline PFOS & $74(-31,178)$ & 0.167 & $-0.0(-0.4,0.4)$ & 0.987 & $0.2(-0.1,0.4)$ & 0.189 & $-0.01(-0.3,0.3)$ & 0.952 & $0.71(0.42-1.20)$ & 0.201 \\
\hline PCB 153 & $100(-104,304)$ & 0.334 & $-0.1(-1.0,0.7)$ & 0.772 & $0.2(-0.3,0.7)$ & 0.507 & $0.1(-0.5,0.7)$ & 0.693 & $0.70(0.25-1.98)$ & 0.502 \\
\hline$p, p^{\prime}-\mathrm{DDE}$ & $46(-47,139)$ & 0.327 & $0.2(-0.2,0.6)$ & 0.306 & $0.1(-0.2,0.3)$ & 0.531 & $-0.04(-0.3,0.2)$ & 0.747 & $0.73(0.46-1.15)$ & 0.174 \\
\hline $\mathrm{HCB}$ & $61(-132,255)$ & 0.534 & $0.1(-0.7,0.9)$ & 0.792 & $0.1(-0.4,0.6)$ & 0.626 & $-0.1(-0.6,0.5)$ & 0.756 & $0.52(0.20-1.34)$ & 0.176 \\
\hline$B-\mathrm{HCH}$ & $-73(-231,86)$ & 0.368 & $-0.5(-1.1,0.2)$ & 0.152 & $-0.1(-0.5,0.3)$ & 0.614 & $-0.1(-0.6,0.3)$ & 0.592 & $0.76(0.33-1.71)$ & 0.500 \\
\hline$t-\mathrm{NC}$ & $98(-56,253)$ & 0.212 & $0.2(-0.5,0.8)$ & 0.605 & $0.4(-0.02,0.8)$ & 0.065 & $0.2(-0.2,0.7)$ & 0.383 & $0.86(0.40-1.84)$ & 0.699 \\
\hline \multicolumn{11}{|l|}{ Sweden } \\
\hline PFOA & $-359(-596,-122)$ & 0.003 & $-1.3(-2.3,-0.3)$ & 0.010 & $-0.4(-1.0,0.1)$ & 0.115 & $-0.3(-0.9,0.3)$ & 0.318 & $5.25(1.68-16.4)$ & 0.004 \\
\hline PFOS & $-292(-500,-84)$ & 0.006 & $-1.2(-2.1,-0.3)$ & 0.007 & $-0.4(-0.9,0.04)$ & 0.073 & $-0.4(-0.9,0.2)$ & 0.201 & $2.51(0.93-6.77)$ & 0.068 \\
\hline PCB 153 & $-11(-374,352)$ & 0.953 & $0.1(-1.4,1.6)$ & 0.891 & $-0.2(-1.1,0.6)$ & 0.558 & $0.6(-0.4,1.6)$ & 0.222 & $5.59(1.05-29.9)$ & 0.044 \\
\hline$p, p^{\prime}-\mathrm{DDE}$ & $38(-147,223)$ & 0.688 & $0.3(-0.4,1.1)$ & 0.400 & $0.2(-0.2,0.7)$ & 0.273 & $0.4(-0.04,0.9)$ & 0.072 & $1.70(0.75-3.85)$ & 0.200 \\
\hline $\mathrm{HCB}$ & $-269(-595,57)$ & 0.105 & $-1.0(-2.4,0.3)$ & 0.134 & $-1.0(-1.7,-0.2)$ & 0.011 & $0.1(-0.7,1.0)$ & 0.742 & $5.62(1.26-25.1)$ & 0.024 \\
\hline $\mathrm{B}-\mathrm{HCH}$ & $-161(-446,125)$ & 0.268 & $-0.4(-1.6,0.8)$ & 0.537 & $-0.4(-1.1,0.2)$ & 0.209 & $0.1(-0.7,0.8)$ & 0.852 & $3.20(0.84-12.1)$ & 0.087 \\
\hline$t-\mathrm{NC}$ & $-92(-379,195)$ & 0.529 & $-0.2(-1.4,1.0)$ & 0.766 & $-0.4(-1.1,0.2)$ & 0.213 & $0.3(-0.5,1.1)$ & 0.452 & $2.04(0.64-6.55)$ & 0.229 \\
\hline
\end{tabular}

${ }^{a}$ Adjusted for maternal age (years), height ( $\left.\mathrm{cm}\right)$, prepregnancy BMI ( $\left.\mathrm{kg} / \mathrm{m}^{2}\right)$, education $(<9,9-12,>12 \mathrm{y})$, parity $(1$ or 2$)$, smoking status at conception $(0,1-9,>10$ cig/d), interpregnancy

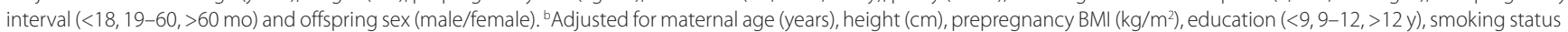
at conception $(0,1-9,>10 \mathrm{cig} / \mathrm{d})$, and interpregnancy interval $(<18,19-60,>60 \mathrm{mo})$.

HCB, hexachlorobenzene; PCB, polychlorinated biphenyl; PFOA, perfluorooctanoate; PFOS, perfluoroctane sulfonate.

Table 4. Beta coefficients $(\beta)$ and odds ratios (ORs) with $95 \%$ confidence intervals $(95 \% \mathrm{Cl})$ for associations between PFOA and indices of fetal growth in Sweden by offspring sex $(N=159)$

\begin{tabular}{|c|c|c|c|c|c|c|c|c|c|c|c|}
\hline & & \multicolumn{2}{|c|}{ Birth weight $(\mathrm{g})^{\mathrm{a}}$} & \multicolumn{2}{|c|}{ Birth length $(\mathrm{cm})^{\mathrm{a}}$} & \multicolumn{2}{|c|}{ Head circumference ${ }^{a}(\mathrm{~cm})$} & \multicolumn{2}{|c|}{ Gestational age (weeks) } & \multicolumn{2}{|l|}{$S G A^{b}$} \\
\hline & & $\beta(95 \% \mathrm{Cl})$ & $P$ & $\beta(95 \% \mathrm{Cl})$ & $P$ & $\beta(95 \% \mathrm{Cl})$ & $P$ & $\beta(95 \% \mathrm{Cl})$ & $P$ & OR $(95 \% \mathrm{Cl})$ & $P$ \\
\hline PFOA & $N$ & & & & & & & & & & \\
\hline Boys & 81 & $-526(-828,-222)$ & 0.001 & $-1.6(-2.9,-0.4)$ & 0.012 & $-0.6(-1.3,0.1)$ & 0.103 & $-0.4(-1.2,0.5)$ & 0.365 & $6.55(1.14-37.45)$ & 0.035 \\
\hline Girls & 78 & $-156(-541,228)$ & 0.419 & $-0.8(-2.4,0.8)$ & 0.340 & $-0.1(-1.0,0.7)$ & 0.728 & $-0.1(-1.1,0.9)$ & 0.802 & $4.73(0.79-28.3)$ & 0.089 \\
\hline
\end{tabular}

${ }^{a}$ Adjusted for maternal age (years), height ( $\left.\mathrm{cm}\right)$, prepregnancy BMI (kg/m²), education $(<9,9-12,>12 \mathrm{y})$, parity $(1$ or 2$)$, smoking status at conception $(0,1-9,>10$ cig/d), inter-pregnancy interval $(<18,19-60,>60 \mathrm{mo})$, and offspring sex (male/female). $\left.{ }^{\text {b} A d j u s t e d ~ f o r ~ m a t e r n a l ~ a g e ~(y e a r s), ~ h e i g h t ~(~} \mathrm{cm}\right)$, prepregnancy BMl (kg/m²), education ( $\left.<9,9-12,>12 \mathrm{y}\right)$, smoking status at conception $(0,1-9,>10 \mathrm{cig} / \mathrm{d})$, and interpregnancy interval $(<18,19-60,>60 \mathrm{mo})$.

PFOA, perfluorooctanoate.

for some differences between populations. Although we did not find evidence of effect modification by smoking in our study, the high proportion of smokers in our study population may introduce bias and thereby underestimate true effects of the contaminants. In addition, due to the strong associations between smoking and fetal growth, the true effects of EDCs could be overshadowed and hard to detect, even in adjusted analyses.

Assessing potential causal effects of multiple EDCs on health outcomes are complicated because of the complex correlation of exposures. Associations found in some studies may largely reflect correlated EDC exposures instead of a specific EDC under study. In our study, we found moderate or high correlations within the PFASs $(\rho=0.56-0.73)$ and within the OCs $(\rho=0.35-0.70)$ (Supplementary Figure S1 online), but only weak correlations between the PFASs and
OCs $(\rho=-0.01-0.22)$. The lack of correlation between PFASs and OCs made it possible to distinguish between the independent effects of PFASs and OCs. When assessing individual EDCs, we demonstrated positive associations between PFOA, PCB 153 and HCB and SGA birth (Table 3). However, the only association that remained statistically significant when including the other contaminants as covariates in final models was the association between PFOA and SGA birth (Supplementary Table S3 online). This further confirms that the association between PFOA and SGA birth is not explained by correlated exposures. The association between HCB and SGA birth was also significant when we adjusted for PFOA. Based on these analyses, it seems like both PFOA and HCB are independently associated with SGA birth. Accounting for correlations within and between different EDCs strengthens our findings by distinguishing between contaminants 
a

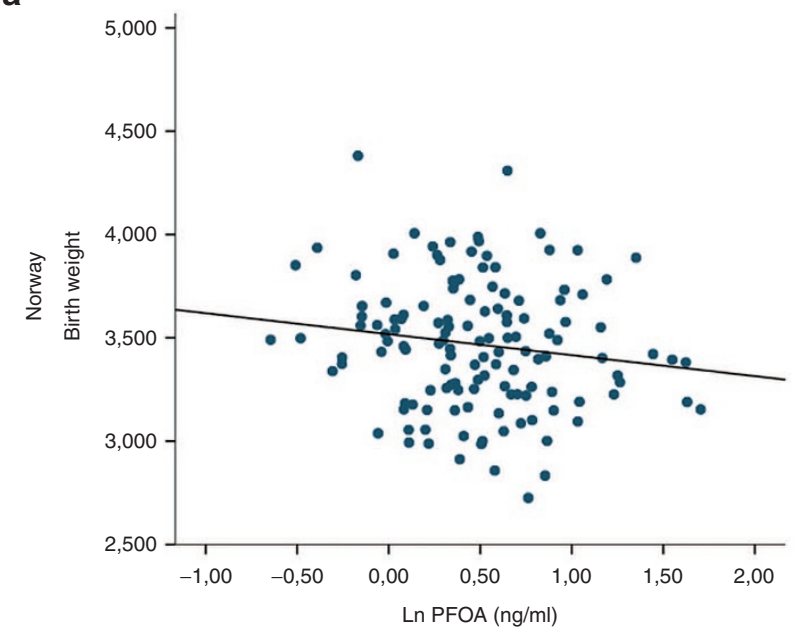

C

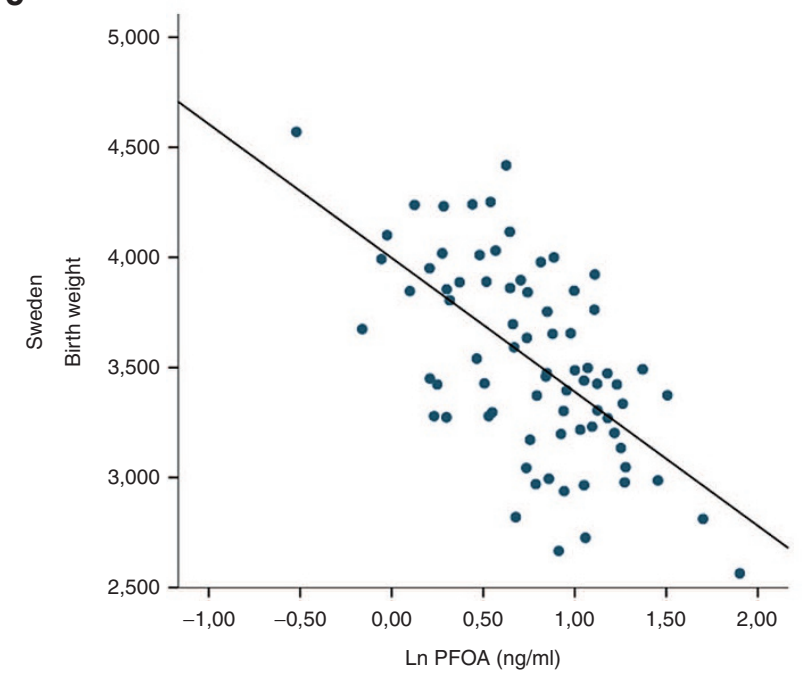

b

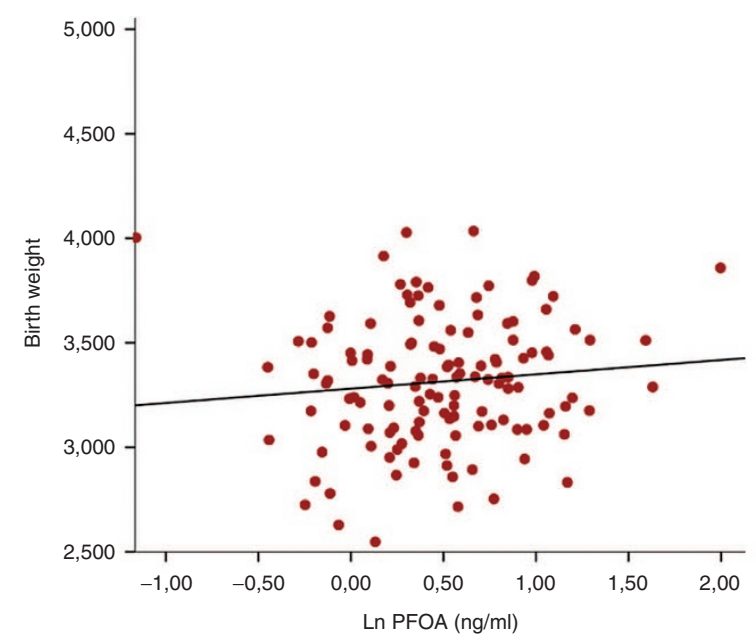

d

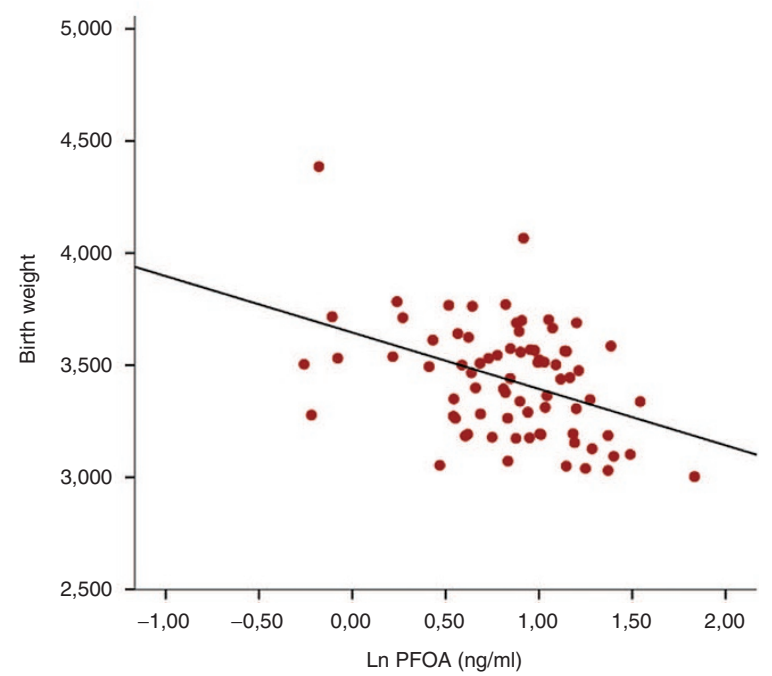

Figure 2. Scatter plot with $\ln (\mathrm{PFOA}(\mathrm{ng} / \mathrm{ml})$ ) values on the $\mathrm{X}$-axis and adjusted values of birth weight $(\mathrm{g})$ on the $\mathrm{Y}$-axis, stratified by country and offspring sex. Blue dots: boys, red dots: girls. (a) Norwegian boys, (b) Norwegian girls, (c) Swedish boys, (d) Swedish girls. PFOA, perfluorooctanoate.

that may have independent causal effects on restricted fetal growth.

This study has several strengths including a large, homogeneous population of mother-child pairs $(n=424)$. Ours is one of few studies to investigate a variety of PFAS and OC exposures. Since PFASs and OCs were not highly correlated, our results show an independent association between PFOA and fetal growth. The unique design of this case-cohort study accounts for a high proportion of SGA births as a marker for FGR, rather than birth weight alone. Therefore, this study has greater power to detect clinically relevant associations between EDCs and FGR using SGA birth as an outcome. We further tested the generalizability of our results by excluding the women from the high-risk group in all the linear and logistic models, and the associations in the Swedish cohort persisted (data not shown). The use of lipid-adjusted serum levels of OCs may be prone to bias in epidemiological studies (27). We therefore analyzed all linear and logistic models using wet weight values adjusted for total lipids as an independent variable with no considerable change in point estimates (data not shown). Maternal weight gain during pregnancy is suggested to be an important confounder in studies with lipophilic chemicals, but mainly in studies using serum from late pregnancy or cord blood (28). Since maternal weight gain also represents blood volume expansion, it may also be an important confounder in studies with PFASs, which largely are bound to albumin in the blood (4). Alcohol consumption may also be a confounder because it has been associated with higher levels of OCs (29). However, sensitivity analyses revealed that the estimates did not change when we included weight gain or alcohol consumption as covariates in the analyses (Supplementary Tables S4 and S5 online).

This study also has some weaknesses. We have not corrected for multiple comparisons, which increases the chance of a rare event and enables false-positive results (i.e., Type I error). Measures of renal filtration have been proposed to be important confounders in studies with PFASs and fetal growth (30). Unfortunately, we only had glomerular filtration 


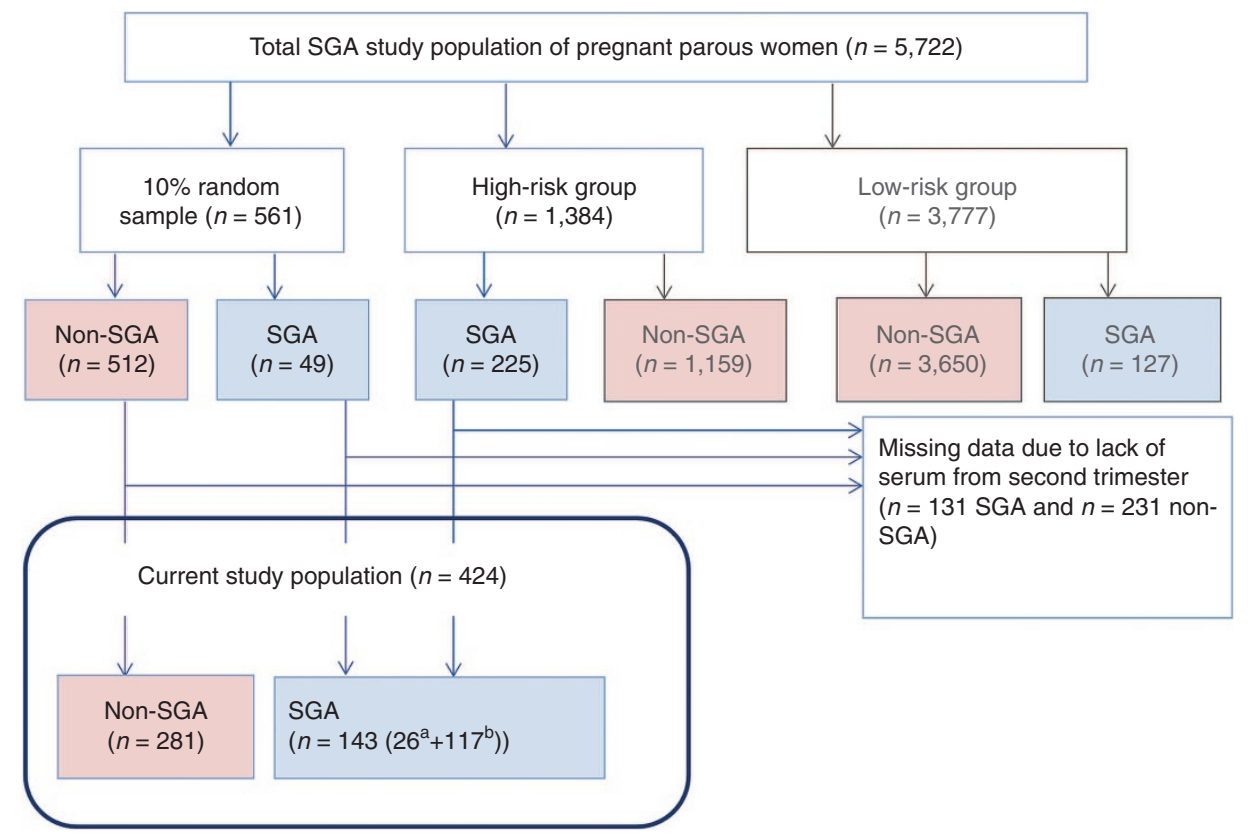

Figure 3. Flow chart of the study selection. a Number of participants from the $10 \%$ random sample. ${ }^{b}$ Number of participants from the high-risk group.

rate (GFR) for 88 women, and could not include GFR as a covariate in adjusted models due to problems with missing data. Primiparous women were not eligible for study inclusion, which may contribute to some selection bias. However, excluding these women may largely reduce confounding due to parity because first time mothers have a higher risk of delivering small babies (31). At the same time, primiparous women may have higher serum EDC levels, because they lack previous excretion through placenta and breastmilk (2). Persistent and bio-accumulative chemicals with the same properties are highly correlated. Therefore, our point estimates may be subject to residual confounding due to some unmeasured chemicals (e.g., lead) in our analyses.

\section{Conclusion}

We found higher odds for SGA birth with increasing serum levels of PFOA, PCB 153 and HCB, but only in the Swedish cohort. Both PFOA and HCB were associated with SGA birth after accounting for correlation between EDCs. We observed stronger associations between increasing levels of PFOA and SGA birth in male offspring from the Swedish cohort. Associations between EDCs and indices of fetal growth in the Norwegian cohort were null. Our results suggest that some populations may be more vulnerable to EDCs, possibly due to differences in EDC levels, exposure sources and/or potentially modifiable lifestyle factors. The study also suggests that male offspring may be more vulnerable to endocrine disruption than female offspring.

\section{METHODS}

\section{Ethics}

The project is approved by the Central Norway Regional Committee for Medical and Health Science Research Ethics (REK Midt 2010/1449-5).

\section{Study Population}

Participants were from the US National Institute of Child Health and Human Development (NICHD) Scandinavian Successive Small-forGestational Age (SGA) births study; a population-based prospective multicenter study conducted in Trondheim and Bergen (Norway) and Uppsala (Sweden). Participant recruitment occurred over a 27-mo period (1986-1988). The SGA births study was designed to study the etiology and consequences of intrauterine growth restriction (32). Since the study also examined the tendency for individual women to repeat an SGA birth outcome in consecutive pregnancies, first time mothers were ineligible for study participation. Eligible participants were second and third time mothers of Caucasian origin who spoke one of the Scandinavian languages, had a singleton pregnancy, and were registered by the study center prior to the 20th gestational week. In total, we recruited 5,722 women, from which we defined three groups: a $10 \%$ random sample representative of the parous population at each study site $(n=561)$; a group at high risk for SGA birth ( $n$ $=1,384)$, and a low risk group $(n=3,777)$ (Figure 3 ). Both the random sample and high-risk group were included for detailed follow-up throughout pregnancy and at birth. The high risk group was defined by one or more of the following risk factors: (i) a prior SGA or lowbirth-weight (LBW) child, (ii) maternal cigarette smoking at conception, (iii) low prepregnancy weight $(<50 \mathrm{~kg}$ ), (iiii) a previous perinatal death, or (iiiii) the presence of chronic maternal disease including essential hypertension or renal disease. In the current study, we used a case-cohort design (33) to estimate the associations between prenatal EDC exposure and indices of fetal growth. In total, 143 SGA births were selected as cases, and 281 non-SGA controls were selected from the $10 \%$ random sample group (total $n=424$ ) (Figure 3). Selection was based on the availability of second trimester maternal serum samples.

\section{Exposure Assessment}

Serum samples were collected in second trimester (gestational week 17-20) in the SGA study (1986-1988) and serum was stored at -80 ${ }^{\circ} \mathrm{C}$ for later analysis. All 424 samples were included for PFAS and OC analyses. All chemicals presented were detected in $100 \%$ of the samples, except $\beta$-hexachlorohexane $(\beta-\mathrm{HCH})$ that was detected in $99 \%$. Limits of detection (LODs) are listed in Table 2, and values below LOD were replaced by LOD $/ \sqrt{ } 2$.

\section{Chemical Analyses of PFAS}

Analyses were performed at the laboratories of Norwegian Institute for Air Research, Tromsø, Norway (NILU). All serum samples were 


\section{Perfluoroalkyl substances and organochlorines and fetal growth}

quantified for two target analytes; perfluorooctanoate (PFOA) and PFOS. They were analyzed using sonication-facilitated liquid-liquid extraction, activated ENVI-carb clean-up (34), quantified and analyzed by ultrahigh pressure liquid chromatography triple-quadruple mass-spectrometry (UHPLC-MS/MS). Sample preparation and extraction were performed as described by Hanssen et al (35) except for minor volume changes. The quantification was conducted with the LC Quan software, version 2.6.0 (Thermo Fisher Scientific, Waltham, MA). The internal-standard addition method with isotope-labeled PFASs was used to quantify the contaminants (35). Participation in the AMAP Ring Test (36) indicates that the uncertainties of the analysis are within $\pm 15-20 \%$ of the assigned values.

\section{Chemical Analyses of OCs}

OCs were analyzed at the Institut National de Santé Publique du Quebec, Centre Toxicologie, Quebec. This laboratory is the organizer of the AMAP Ring Test (36). OCs measured were hexachlorobenzene (HCB), oxychlordane, polychlorinated biphenyl (PCB) 52, 101, $118,153,156,170$, and $180, p, p$-dichlorodiphenyldichloroehylene $\left(p, p^{\prime}\right.$-DDE $), \quad p, p^{\prime}$-dichlorophenyltrichloroethane $\left(p, p^{\prime}\right.$-DDT $), \quad \beta$ hexachlorohexane $(\beta-\mathrm{HCH})$ and trans-nonachlor $(t-\mathrm{NC})$. We chose to report PCB 153 representing total PCBs, and excluded $p, p$-DDT because of low detection limit ( $>50 \%$ of samples $<\mathrm{LOD})$. In short, $0.5-1 \mathrm{ml}$ serum sample was extracted using hexane $(2 \times 6 \mathrm{ml})$, ethanol $(2 \mathrm{ml})$ and saturated ammonium sulphate solution $(2 \mathrm{ml})$, a slight modification of Sandanger et al. (37).

\section{Outcome Assessment}

Indices of fetal growth including birth weight (continuous; grams $(\mathrm{g})$ ), birth length (continuous; centimeters $(\mathrm{cm})$ ) and head circumference (continuous; $\mathrm{cm}$ ) were measured and recorded at birth. Gestational age (continuous; completed weeks) was determined by ultrasound scan at $17 \mathrm{wk}$ of gestation. SGA birth was defined as birth weight below the 10th percentile adjusted for gestational age, parity and sex of child (32).

\section{Covariates}

Based on prior knowledge of PFAS and OC properties, and known risk factors for SGA birth, we included potential confounders from data collected at first study visit in gestational week 17 . These involved maternal age (continuous; years), maternal height (continuous; $\mathrm{cm}$ ), maternal prepregnancy BMI (continuous; $\mathrm{kg} / \mathrm{m}^{2}$ ), education level (categorical; 9 y or less, 10-12 y, or 13 y or more), smoking status at conception (categorical; $0,1-9$ or $>10$ cigarettes per day), parity (binary; 1 or 2) and interpregnancy interval (categorical; 18 mo or less, 19-60 mo, $61 \mathrm{mo}$ or more). We categorized the interpregnancy interval based on a known J-shaped association to adverse perinatal outcomes including restricted fetal growth (38). Offspring sex (male/ female) was registered at birth. Weight gain up to $17 \mathrm{wk}$ was calculated based on recorded weight measurements done by midwives at the regular prenatal visits throughout pregnancy. Alcohol consumption was self-reported in gestational week 33. Dietary information from the Norwegian women consisted of a self-reported food frequency questionnaire over a 3-d period in week 17 and 33.

\section{Statistical Analyses}

PFAS and OC levels were logarithmically (ln) transformed to obtain normal distribution. We used wet weight concentrations of PFAS and lipid-adjusted serum concentrations of OCs (39). Total lipid values were calculated based on measurements of triglycerides and cholesterol:

Total lipids $=1.33^{*}$ triglycerides $+1.12^{\star}$ cholesterol $+1.48(\mathrm{~g} / \mathrm{l})$ (39). This formula showed good correlation with complete formulas including phospholipids (40).

In a complete case analysis we used uni- and multivariate linear regression with $95 \%$ confidence intervals (CIs) to estimate the adjusted associations between natural log-transformed (ln) serum levels of seven individual EDCs (PFASs: PFOA, PFOS; OCs: PCB153, p,p'$\mathrm{DDE}, \mathrm{HCB}, t-\mathrm{NC}$, and $\beta$-HCH) and indices of fetal growth including birth weight, birth length, head circumference and gestational age at birth. We evaluated linear model assumptions using diagnostic plots of the residuals. In all linear regression analyses we adjusted for the same covariates (maternal age, maternal height, prepregnancy BMI, maternal education level, smoking at conception, parity, interpregnancy interval, and offspring sex). We conducted uni- and multivariate logistic regression to estimate the crude and adjusted odds ratios (ORs and aORs) for SGA birth per unit increase in natural log-transformed (ln) serum levels of each PFAS and OC. In all the logistic regression analyses we adjusted for the same covariates (maternal age, maternal height, prepregnancy BMI, maternal education level, smoking at conception, and interpregnancy interval). SGA birth was defined as birthweight below the 10th percentile adjusted for gestational age, parity and offspring sex; hence, we did not adjust for parity or offspring sex in the models with SGA birth as an outcome. Based on the evidence of effect modification by geography, we stratified our results by country of residence. In multivariate logistic models with SGA as outcome variable, we found significant $P$ values for interaction between the different EDCs and country of residence (PFOA $P=0.043$; PCB $153 P=0.073$; HCB $P=0.008$ ). In multivariate linear regression models with birth weight as an outcome, $P$ values for the interaction term were $0.004,0.001$, and 0.036 for PFOA, PFOS, and HCB, respectively. Within country-specific strata, we further considered possible effect modification by offspring sex, because proposed endocrine disrupting properties might be sex-specific. Missing data was less than $10 \%$ for included covariates. All statistical analyses were conducted with SPSS statistical software, version 22 (IBM SPSS, Chicago, IL).

\section{SUPPLEMENTARY MATERIAL}

Supplementary material is linked to the online version of the paper at http:// www.nature.com/pr

\section{ACKNOWLEDGMENTS}

We gratefully acknowledge the participating women in the SGA-study. We would like to thank people at NILU - Norwegian Institute of Air Research in Tromsø, Norway, for conducting the PFAS analyses, and Institut National de Santé Publique du Québec, Centre de Toxicologie in Quebec, for the OC analyses. Special thanks to Charlotta Rylander, Therese H. Nøst, and Vivian Berg, for valuable input and advice.

\section{STATEMENT OF FINANCIAL SUPPORT}

This work was funded by grants from the Liaison Committee between the Central Norway Regional Health Authority and the Norwegian University of Science and Technology.

Disclosure: The authors have nothing to disclose.

\section{REFERENCES}

1. Wollmann HA. Intrauterine growth restriction: definition and etiology. Horm Res 1998;49 Suppl 2:1-6.

2. Barr DB, Bishop A, Needham LL. Concentrations of xenobiotic chemicals in the maternal-fetal unit. Reprod Toxicol 2007;23:260-6.

3. World Health Organization UNEP. State of the Science of Endocrine Disruptive Chemicals, Summary for Decision-Makers, 2012. (http://apps. who.int/iris/bitstream/10665/78102/1/WHO_HSE_PHE_IHE_2013.1_ eng.pdf?ua=1).

4. Bach CC, Bech BH, Brix N, Nohr EA, Bonde JP, Henriksen TB. Perfluoroalkyl and polyfluoroalkyl substances and human fetal growth: a systematic review. Crit Rev Toxicol 2015;45:53-67.

5. Govarts E, Nieuwenhuijsen M, Schoeters G, et al.; OBELIX; ENRIECO. Birth weight and prenatal exposure to polychlorinated biphenyls (PCBs) and dichlorodiphenyldichloroethylene (DDE): a meta-analysis within 12 European Birth Cohorts. Environ Health Perspect 2012;120:162-70.

6. Vestergren R, Berger U, Glynn A, Cousins IT. Dietary exposure to perfluoroalkyl acids for the Swedish population in 1999, 2005 and 2010. Environ Int 2012;49:120-7.

7. Bjermo H, Darnerud PO, Pearson M, et al. Serum concentrations of perfluorinated alkyl acids and their associations with diet and personal characteristics among Swedish adults. Mol Nutr Food Res 2013;57:2206-15.

8. Domingo JL, Bocio A. Levels of PCDD/PCDFs and PCBs in edible marine species and human intake: a literature review. Environ Int 2007;33:397-405.

9. Nyberg E, Faxneld S, Danielsson S, Eriksson U, Miller A, Bignert A. Temporal and spatial trends of PCBs, DDTs, HCHs, and HCB in Swedish marine biota 1969-2012. Ambio 2015;44 Suppl 3:484-97. 
10. Rylander L, Strömberg U, Hagmar L. Decreased birthweight among infants born to women with a high dietary intake of fish contaminated with persistent organochlorine compounds. Scand J Work Environ Health 1995;21:368-75.

11. Skåre JU, Brantsaeter AL, Frøyland L, et al. Benefit-risk assessment of fish and fish products in the Norwegian diet - an update. European J Nutr Food Saf 2015;5:260-266.

12. Wilcox AJ, Weinberg CR, Basso O. On the pitfalls of adjusting for gestational age at birth. Am J Epidemiol 2011;174:1062-8.

13. Chen $\mathrm{MH}, \mathrm{Ha} \mathrm{EH}$, Wen TW, et al. Perfluorinated compounds in umbilical cord blood and adverse birth outcomes. PLoS One 2012;7:e42474.

14. Whitworth KW, Haug LS, Baird DD, et al. Perfluorinated compounds in relation to birth weight in the Norwegian Mother and Child Cohort Study. Am J Epidemiol 2012;175:1209-16.

15. Hamm MP, Cherry NM, Chan E, Martin JW, Burstyn I. Maternal exposure to perfluorinated acids and fetal growth. J Expo Sci Environ Epidemiol 2010;20:589-97.

16. Fei C, McLaughlin JK, Tarone RE, Olsen J. Perfluorinated chemicals and fetal growth: a study within the Danish National Birth Cohort. Environ Health Perspect 2007;115:1677-82.

17. Blazer S, Moreh-Waterman Y, Miller-Lotan R, Tamir A, Hochberg Z. Maternal hypothyroidism may affect fetal growth and neonatal thyroid function. Obstet Gynecol 2003;102:232-41.

18. Kaijser M, Granath F, Jacobsen G, Cnattingius S, Ekbom A. Maternal pregnancy estriol levels in relation to anamnestic and fetal anthropometric data. Epidemiology 2000;11:315-9.

19. Berg V, Nøst TH, Hansen S, et al. Assessing the relationship between perfluoroalkyl substances, thyroid hormones and binding proteins in pregnant women; a longitudinal mixed effects approach. Environ Int 2015;77:63-9.

20. Brouwer A, Longnecker MP, Birnbaum LS, et al. Characterization of potential endocrine-related health effects at low-dose levels of exposure to PCBs. Environ Health Perspect 1999;107 Suppl 4:639-49.

21. Hamers T, Kamstra JH, Cenijn $\mathrm{PH}$, et al. In vitro toxicity profiling of ultrapure non-dioxin-like polychlorinated biphenyl congeners and their relative toxic contribution to PCB mixtures in humans. Toxicol Sci 2011;121: 88-100.

22. Kjeldsen LS, Bonefeld-Jørgensen EC. Perfluorinated compounds affect the function of sex hormone receptors. Environ Sci Pollut Res Int 2013;20:8031-44.

23. Washino N, Saijo Y, Sasaki S, et al. Correlations between prenatal exposure to perfluorinated chemicals and reduced fetal growth. Environ Health Perspect 2009;117:660-7.

24. Welch AA, Lund E, Amiano P, et al. Variability of fish consumption within the 10 European countries participating in the European Investigation into Cancer and Nutrition (EPIC) study. Public Health Nutr 2002;5(6B): 1273-85.

25. Rylander L, Strömberg U, Dyremark E, Ostman C, Nilsson-Ehle P, Hagmar L. Polychlorinated biphenyls in blood plasma among Swedish female fish consumers in relation to low birth weight. Am J Epidemiol 1998;147:493-502.

26. Gyllenhammar I, Berger U, Sundström M, et al. Influence of contaminated drinking water on perfluoroalkyl acid levels in human serum-A case study from Uppsala, Sweden. Environ Res 2015;140:673-83.

27. Schisterman EF, Whitcomb BW, Louis GM, Louis TA. Lipid adjustment in the analysis of environmental contaminants and human health risks. Environ Health Perspect 2005;113:853-7.

28. Verner MA, McDougall R, Glynn A, Andersen ME, Clewell HJ 3rd, Longnecker MP. Is the relationship between prenatal exposure to PCB-153 and decreased birth weight attributable to pharmacokinetics? Environ Health Perspect 2013;121:1219-24.

29. Rogan WJ, Gladen BC, McKinney JD, et al. Polychlorinated biphenyls (PCBs) and dichlorodiphenyl dichloroethene (DDE) in human milk: effects of maternal factors and previous lactation. Am J Public Health 1986;76:172-7.

30. Verner MA, Loccisano AE, Morken NH, et al. Associations of perfluoroalkyl substances (PFAS) with lower birth weight: an evaluation of potential confounding by glomerular filtration rate using a physiologically based pharmacokinetic model (PBPK). Environ Health Perspect 2015;123: 1317-24.

31. Wilcox MA, Chang AM, Johnson IR. The effects of parity on birthweight using successive pregnancies. Acta Obstet Gynecol Scand 1996;75:459-3.

32. Bakketeig LS, Jacobsen G, Hoffman HJ, et al. Pre-pregnancy risk factors of small-for-gestational age births among parous women in Scandinavia. Acta Obstet Gynecol Scand 1993;72:273-9.

33. Prentice RL A case-cohort design for epidemiologic cohort studies and disease prevention trials. Biometrika 1986;73:1-11.

34. Powley CR, George SW, Ryan TW, Buck RC. Matrix effect-free analytical methods for determination of perfluorinated carboxylic acids in environmental matrixes. Anal Chem 2005;77:6353-8.

35. Hanssen L, Dudarev AA, Huber S, Odland JØ, Nieboer E, Sandanger TM. Partition of perfluoroalkyl substances (PFASs) in whole blood and plasma, assessed in maternal and umbilical cord samples from inhabitants of arctic Russia and Uzbekistan. Sci Total Environ 2013;447:430-7.

36. Institut national de santé publique Q. AMAP: AMAP Ring Test for Persistent Organic Pollutants in Human Serum, 2014. (https://www.inspq.qc.ca/ en/ctq/eqas/amap/description).

37. Sandanger TM, Brustad M, Odland JO, et al. Human plasma levels of POPs, and diet among native people from Uelen, Chukotka. J Environ Monit 2003;5:689-96.

38. Conde-Agudelo A, Rosas-Bermúdez A, Kafury-Goeta AC. Birth spacing and risk of adverse perinatal outcomes: a meta-analysis. JAMA 2006;295:1809-23.

39. Covaci A, Voorspoels S, Thomsen C, van Bavel B, Neels H. Evaluation of total lipids using enzymatic methods for the normalization of persistent organic pollutant levels in serum. Sci Total Environ 2006;366:361-6.

40. Phillips DL, Pirkle JL, Burse VW, Bernert JT Jr, Henderson LO, Needham LL. Chlorinated hydrocarbon levels in human serum: effects of fasting and feeding. Arch Environ Contam Toxicol 1989;18:495-500.

This work is licensed under a Creative Commons Attribution-NonCommercial-NoDerivs $\quad 4.0$ International License. The images or other third party material in this article are included in the article's Creative Commons license, unless indicated otherwise in the credit line; if the material is not included under the Creative Commons license, users will need to obtain permission from the license holder to reproduce the material. To view a copy of this license, visit http://creativecommons.org/ licenses/by-nc-nd/4.0/

(c) The Author(s) (2016) 